\title{
Augmenting Intraoperative 3D Ultrasound with Preoperative Models for Navigation in Liver Surgery
}

\author{
Thomas Lange ${ }^{1}$, Sebastian Eulenstein ${ }^{1}$, Michael Hünerbein ${ }^{1}$, Hans Lamecker ${ }^{2}$, \\ and Peter-Michael Schlag ${ }^{1}$ \\ 1 Department of Surgery and Surgical Oncology \\ Charité - Universitary Medicine Berlin, Germany \\ lange_t@rrk. charite-buch.de \\ 2 Zuse Institute Berlin, Germany
}

\begin{abstract}
Organ deformation between preoperative image data and the patient in the OR is the main obstacle for using surgical navigation systems in liver surgery. Our approach is to provide accurate navigation via intraoperative 3D ultrasound. These ultrasound data are augmented with preoperative anatomical models and planning data as an important additional orientation aid for the surgeon. We present an overview of the whole ultrasound navigation system as well as an approach for fast intraoperative non-rigid registration of the preoperative models to the ultrasound volume. The registration method is based on the vessel center lines and consists of a combination of the Iterative Closest Point algorithm and multilevel B-Splines. Quantitative results for three different patients are presented.
\end{abstract}

\section{Introduction}

The resection of tumors from the liver is a demanding and risky surgical intervention. The exact intraoperative location of the tumor, its relative position to important liver vessels and the boundaries of vascular territories would be a benificial support for precise and safe liver surgery. This support can be provided by a 3D ultrasound-based navigation system, like the SonoWand-System [1] or our system [2. Such systems show the position of surgical instruments in relation to an intraoperative ultrasound volume. The advantage of an ultrasound based system is that it is inexpensive and can be integrated easily into the OR. One of the limitations of intraoperative 3D ultrasound is image quality such that tumor and vessels are sometimes difficulty to delineate. Transmission of models of portal veins, hepatic veins, tumor and liver surface from preoperative CT/MR scans onto the ultrasound images can significantly improve differentiation of these structures. The relation of ultrasound planes to preoperative data or models would increase the orientation ability of the surgeon. In addition the transmission of a preoperative resection plan to the patient in the OR is possible. Several systems have been developed for liver surgery planning in the last couple of years $[3,4,5]$. But the precise implementation of the plan in the OR is still an 
open problem. In oncological liver surgery the aim is to completely resect one or several lesions with a security margin and to resect as little healthy parenchyma as possible. In most cases however also healthy parenchyma has to be resected if its blood supply and drainage would be disrupted by the surgery. The purpose of the planning systems is to compute anatomical resection proposals based on the vascular territories as shown in Fig. 1 a) and b).

For transmission of models and resection plans to the patient in the OR it is necessary to register preoperative and intraoperative image data. In contrast to neurosurgery or orthopedic surgery rigid registration via landmarks or surfacematching of bony structures is not possible due to significant organ deformations. Since fast and precise automatic algorithms for non-rigid intraoperative registration of 3D ultrasound data are still under development, for neurosurgery Lindseth et al. [6] suggested only to rigidly register preoperative data, trust on ultrasound navigation and use the preoperative data as an orientation aid. Applications in abdominal interventions which make use of preoperative image data to augment navigated intraoperative ultrasound scans include: laparoscopy 7 8] for better orientation and thermal ablation of liver lesions [9]10] for precise placement of preplanned applicator positions.

Fast and robust intraoperative registration is the crucial task for augmenting the ultrasound data. Hence automated rigid and non-rigid registration methods, that have been applied or adapted to 3D ultrasound data, are reviewed in the following. Some image-based methods [111210] have been reported to rigidly register 3D ultrasound and MR data. Non-rigid image-based algorithms for registration of two ultrasound volumes are described in 1314. These approaches are usually too time-consuming for intraoperative use. Liver vessels are features which can be easily identified in $\mathrm{CT} / \mathrm{MR}$ and ultrasound data, in particular in Powerdoppler ultrasound. A feature-based rigid approach using correlation between segmented vessel voxels is reported in [15. In [16] manually identified vessel center lines are rigidly registered via the Iterative Closest Point (ICP) algorithm. Hybrid approaches, which fit preoperatively extracted features directly to intraoperative image data, are promising. The lack of a time constraint in the preoperative phase allows for precise feature extraction yielding fast intraoperative registration. Aylward et al. [17] use vessel models and a special metric for rigid hybrid registration.

We follow the approach to use intraoperative 3D ultrasound for precise navigation and augment it with preoperative data and models. For fast intraoperative non-rigid registration we combine the ICP algorithm and multilevel B-Splines, as in 18 . In contrast to Xie et al. the correspondence determination is not based on surface similarity but on vessel center line points in both modalities.

\section{Methods}

Preoperatively liver parenchyma, portal veins, hepatic veins and the tumor are segmented from MR/CT data. Portal veins and hepatic veins are imaged in two different acquisitions, because their contrast maximum is reached at different times after contrast agent application. Parts of the portal veins are also imaged in the hepatic vein phase so that they can be registered with our vessel-based 

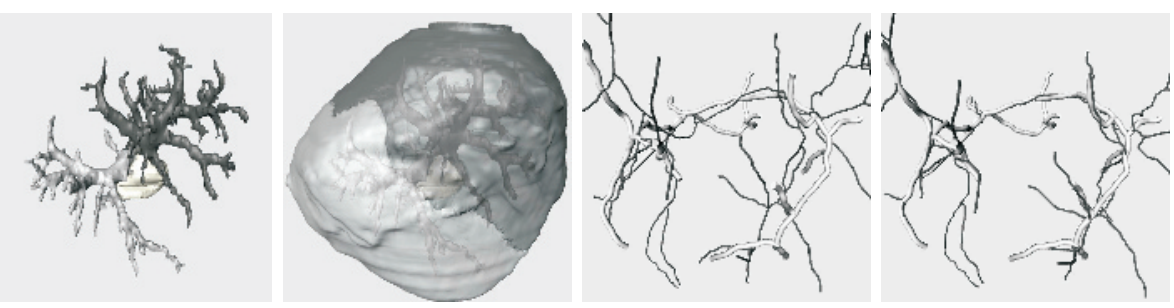

Fig. 1. (a) Portal veins devided into to be resected (light gray) and remaining vessels (dark gray) resulting from tumor location (white). (b) Resulting vascular territories of liver parenchyma. (c) Rigidly and (d) non-rigidly registered portal vein center lines from MR/CT (thin and dark) and 3D US (thick and bright).

non-rigid registration algorithm to get a joint representation of all vessels. Afterwards a resection proposal is automatically computed by our planning software based on the segmented structures. These preoperative models and the resection proposal are transfered to the ultrasound navigation system. In the following we give a short overview of our navigation system, a description of the non-rigid registration procedure and suitable intraoperative visualization methods.

\subsection{US Navigation System}

In contrast to freehand 3D ultrasound systems, where the volume is compounded from manually moved and tracked 2D ultrasound data, we use the 3D ultrasound device Voluson 730D from Kretztechnik/GE. This system is based on a 3D probe containing a 2D ultrasound transducer, which is mechanically swept by a motor. The advantage of this system is that it is fast and can easily be applied in the OR. Intraoperatively a 3D ultrasound scan consisting of simultaneous B-Mode and Powerdoppler (PD) acquisition is performed in a few seconds. The position of a passive tracker attached to the ultrasound probe is measured during the acquisition by a Polaris tracking system. The data are digitally transfered in high quality to the navigation system via a DICOM interface and not via the video output. Afterwards tracked surgical instruments can be navigated in relation to the 3D ultrasound data. For a more detailed description of the ultrasound navigation system see [2].

\subsection{Registration Based on Vessel Center Lines}

Before surgery the vessels are segmented from preoperative CT or MR by a region growing algorithm and manual post-processing to assure the segmentation of as many vessels as possible. Next the center lines of the vessels are automatically extracted by the TEASAR algorithm [19]. The intraoperative pre-processing starts with a reformatting of the 3D US data to Cartesian coordinates, because of the specific original imaging geometry. After this reformatting the center lines of the vessels are extracted from the PD US volume like they were extracted from the preoperative data. The first step of the registration procedure is a coarse rigid 

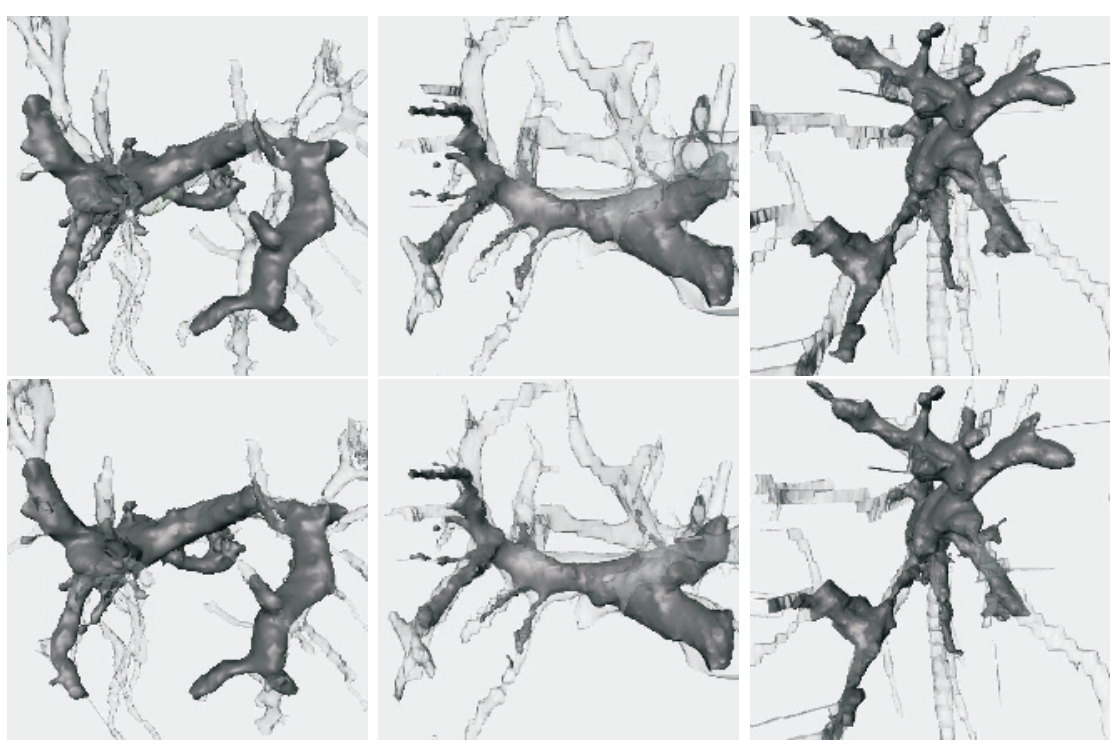

Fig. 2. Rigidly (upper row) and non-rigidly (lower row) registered vessel surfaces from $\mathrm{MR} / \mathrm{CT}$ data (transparent) to 3D US (opaque) of three different patients (from left too right).

registration of the center lines via 3-4 manually selected paired landmarks near the main branching of the portal vein. The second step consists of an automatic ICP-like rigid registration. In the third step non-rigid transformations modeled by multilevel B-Splines are incorporated into the ICP-like registration.

In contrast to the standard ICP algorithm in each iteration corresponding vessel center line points of reference and model data are determined instead of corresponding points between surfaces. Because of the branching topology of vessels the nearest point of a model point to the reference center lines often does not correspond anatomically. Thus we search for the closest vessel segment with a similar direction. A vessel segment is to be defined as a part of the center line between two branching points. For each point $M$ on the model those vessel segments $S_{i}$ in the reference are sought for, which have a closest point $C_{i}$ to $M$ that is inside a given search radius $R$. All the potential corresponding segments $S_{i}$ are sorted by increasing distance of $M$ to $C_{i}$. Starting with $S_{1}$ the closest segment $S_{c}$ is determined for which the angular difference of the vessel direction at $M$ and $C_{i}$ is below a given threshold. The direction at a vessel point is computed by the difference vector of the two neighbored points on the vessel. To increase robustness we averaged the directions of 5 neighboring points. If no corresponding segments can be found that fulfill maximal distance $(R=10 \mathrm{~mm})$ and maximal angle difference $\left(30^{\circ}\right)$ constraints no correspondence is introduced for this model point $M$.

If further improvements can be achieved by applying a rigid transformation in an ICP iteration step it is replaced by a B-Spline transformation. A B-Spline 

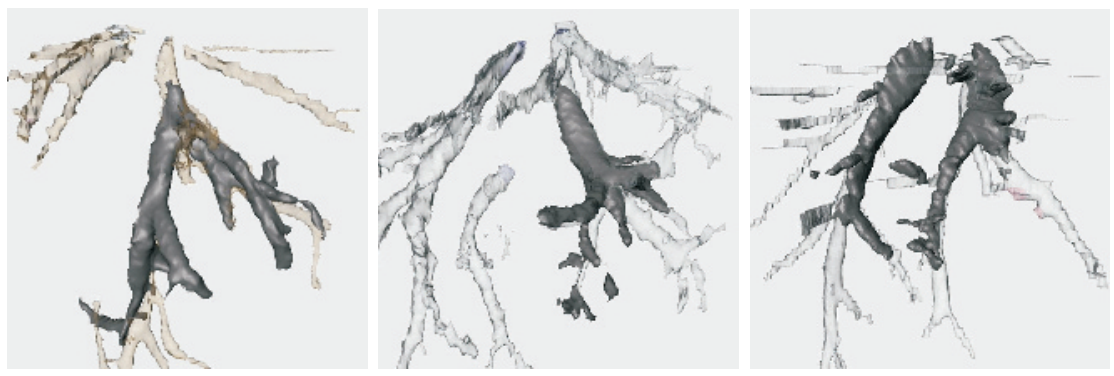

Fig. 3. Non-rigidly registered hepatic veins from CT/MR (transparent) to 3D US (opaque) of three different patients (from left to right). Deformation of hepatic veins is based on B-spline deformation determined by portal vein registration.

approximation of the displacement vectors between corresponding points can be determined directly and fast without need of an optimization algorithm. BSplines are defined by a uniform control grid. Via the control grid spacing it is possible to control the smoothness of the resulting deformations. Finer grids lead to less smoother deformations. Computations of multilevel B-Splines starts with a coarse grid that is successively refined until a given minimal grid spacing is reached. We start the non-rigid ICP iterations with coarse multilevel B-Splines and refine them if no further improvements can be achieved. The minimal control grid spacing has been set to $15 \mathrm{~mm}$.

\subsection{Intraoperative Visualization}

We implemented different intraoperative visualization techniques. For direct US navigation two ultrasound image planes, a top view and a perpendicular slice are shown. The planes can be dynamically chosen according to the position of the tip of the surgical instrument or can be frozen and the instrument is shown in relation to the planes. The registered preoperative models of vessels, tumor, liver surface and resection plan can be rendered as different colored intersection lines into the ultrasound planes. In addition the current position of the surgical instrument and one or both ultrasound planes in relation to the preoperative models are visualized in an extra viewer. It is also possible to show corresponding $\mathrm{CT} / \mathrm{MR}$ slices to the ultrasound planes.

\section{Results}

We performed the registration algorithm retrospectively on data sets of three different patients. The preoperative CT or MR data were acquired during breathhold using contrast agents. Patient 1 and 3 got preoperative T1-weighted Flash 3D VIBE MR-sequences with $2.5 \mathrm{~mm}$ slice thickness. For Patient 2 a single slice spiral CT with $2 \mathrm{~mm}$ reconstructed slice thickness (5mm collimation, pitch 1.5) was acquired. 3D B-mode and Powerdoppler ultrasound was simultaneously acquired transcutaneously for patient 1 and intraoperatively for patient 2 and 
3 using a $3.5 \mathrm{MHz}$ abdominal 3D probe. The original resolution of the powerdoppler scans was $0.2 \mathrm{~mm}$ in scan line direction, $0.5-0.7$ degrees in the scan plane and 0.9 degrees between consecutive scan planes. A scanning volume of approximately 2 liters was reached and usually more than half of the liver is imaged. The original data were resampled isotropically to $1 \mathrm{~mm}$ Cartesian coordinates. Manual selection of 3-4 landmarks near the portal vein trunk for pre-registration lasts 1-2 minutes. The automatic procedures without interactive segmentation of PD-US data and manual pre-registration is possible in 1-2 minutes. The whole registration process lasts less than 15 minutes and can be significantly accelerated by an improved segmentation step, which seems possible.

Correctness and accuracy determination of non-rigid registration algorithms is a non-trivial task. On the one hand we evaluated the correct assignment of preand intraoperative portal vein center lines and on the other hand we measured the deviations of structures which have not been involved in the correspondence determination, like hepatic veins, tumor boundary and liver surface. Vessel center line segments were manually assigned and these assignments were compared with the assignments of the algorithm. We observed only two wrong assignments. In Fig. 1 c) and d) rigidly and non-rigidly registered portal vein center lines of patient 1 are shown. A RMS difference of 5.6, 5.7 and $3.4 \mathrm{~mm}$ has been computed between rigidly and non-rigidly registered center line points of the three patients. The resulting portal vein surfaces match well for all three patients as can be seen in Fig. [2 Similarly in Fig. [3 the surfaces of the hepatic veins are shown which have not been used for correspondence determination. Intersections of the preoperative models with the ultrasound data of patient 2 and 3 are shown in Fig. 4. By inspection of these intersections for all three patients we observed 6 to $9 \mathrm{~mm}$ maximal deviation for the vessels, 12 bis $15 \mathrm{~mm}$ for the tumor and 16 to $20 \mathrm{~mm}$ for the liver surface.

To assess the reproducibility of the results the algorithm was run for 50 different starting positions for each patient simulating different manual preregistrations. The starting positions were uniformly distributed in the range of $+/-5 \mathrm{~mm}$ for each of the three translational parameters and in the range of $+/-5$ degrees for each of the three Euler angles. The RMS error of all points on the model center lines between disturbed and undisturbed starting position was in almost all cases below $0.3 \mathrm{~mm}$ RMS error. We had only $4 \%$ failures with RMS errors between 1.7 and $8.3 \mathrm{~mm}$.

\section{Discussion and Conclusion}

First promising results on augmenting intraoperative 3D ultrasound data of three different patients with preoperative models were shown. The use of portal vein center lines as features for a non-rigid registration approach worked well on all three data sets. While the accuracy in the surrounding of vessels which were involved in the correspondence determination is high the accuracy decreases with increasing distance to those vessels. This indicates that the inaccuracies at the liver surface are caused by the deformations and not by the segmentation or skeletonization process. So far we have used only portal veins for registration, because hepatic veins occur in another time period of contrast agent application. 

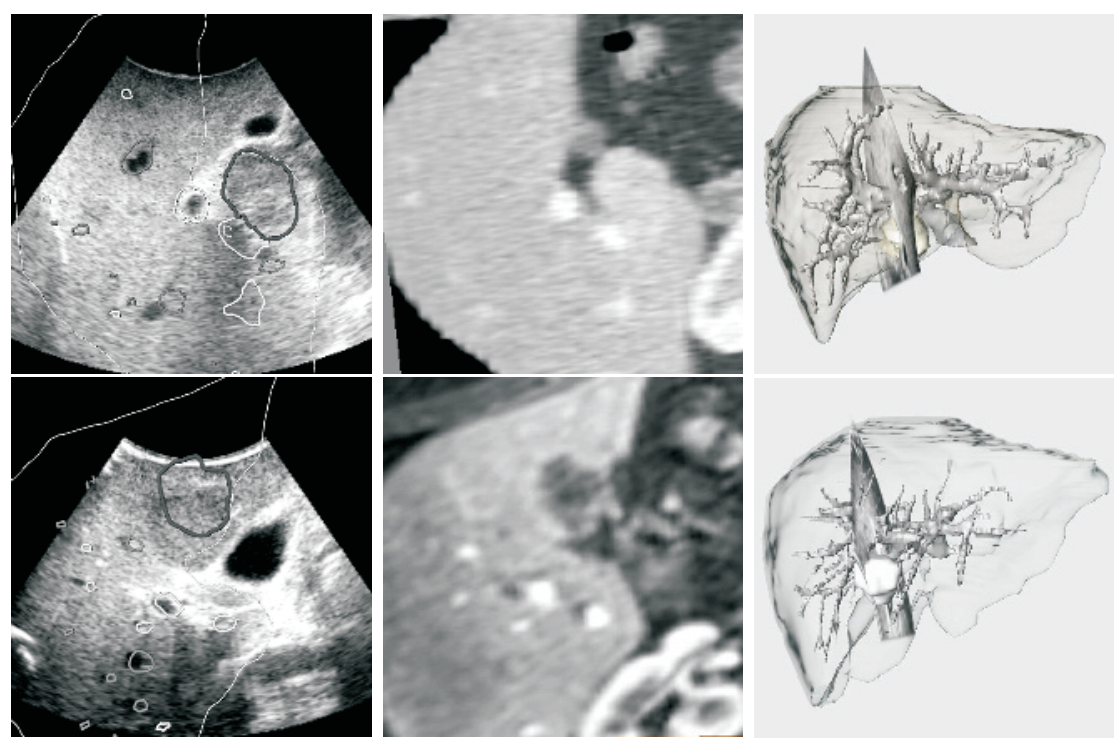

Fig. 4. Intraoperative visualization possibilities for two different patients (upper and lower row). From left to right: US slice with different colored intersection lines of preoperatively modeled tumor (dark and thick), portal veins (bright), hepatic veins (dark) and liver surface (bright and thin). Corresponding CT (upper row) resp. MR (lower row) slice. Overview image showing portal veins, tumor, liver surface and location of US slice.

But we plan to integrate hepatic veins in the future. A possibility to improve the accuracy for structures lying further away from vessels is to incorporate the liver surface into the registration process. Some parts of the liver surface can be identified in the ultrasound images yet other parts can be determined better by a range scanner like in 20 . We will explore both possibilities. We think that for accurate and robust intraoperative liver registration we need both deep lying structures (vessels) and surface information. By streamlining the registration procedure and reducing interaction time for intraoperative vessel segmentation intraoperative non-rigid registration of the liver seems to be possible in less than five minutes.

\section{References}

1. Gronningsaeter A, Kleven A, Ommedal S et al.: SonoWand, an ultrasound-based neuronavigation system. Neurosurgery 47(6):1373-1379, 2000.

2. Eulenstein S, Lange T, Hünerbein M, Schlag PM: Ultrasound based navigation system incorporating preoperative planning for liver surgery. CARS 2004, accepted.

3. Soler L, Delingette H, Malandain G, et al.: Fully automatic anatomical, pathological and functional segmentation from CT scans for hepatic surgery. Computer Aided Surgery 21(11):1344-1357, 2001. 
4. Selle D, Preim B, Schenk A, Peitgen HO: Analysis of vasculature for liver surgical planning. IEEE Trans Med Imaging 21(11):1344-1357, 2002.

5. Meinzer HP, Thorn M, Cardenas C: Computerized planning of liver surgery - an overview. Computers \& Graphics 26(4):569-576, 2002.

6. Lindseth F, Ommedal S, Bang J, et al.: Image Fusion of Ultrasound and MRI as an Aid for assessing Anatomical Shifts and improving overview and interpretation in Ultrasound Guided Neurosurgery. CARS 2001, 1230:247-252, 2001.

7. Kaspersen JH, Sjølie E, Wesche J, et al.: 3D ultrasound based navigation combined with preoperative CT during abdominal interventions: A feasability study. Cardiovasc Intervent Radiol 26:347-356, 2003.

8. Ellsmere J, Stoll J, Rattner D, et al.: A Navigation System for Augmenting Laparoscopic Ultrasound. MICCAI 2003, LNCS 2879:184-191, 2003.

9. Aylward SR, Jomier J, Guyon JP, Weeks S: Intra-Operative 3D Ultrasound Augmentation. IEEE International Symposium on Biomedical Imaging, 2002.

10. Penney GP, Blackall JM, Hamady MS, et al.: Registration of freehand 3D ultrasound and magnetic resonance liver images. Med Image Anal 8(1):81-91, 2004.

11. Roche A, Pennec X, Malandain G, Ayache N: Rigid Registration of 3-D Ultrasound With MR Images: A New Approach Combining Intensity and Gradient Information. IEEE Trans Med Imag 20(10):1038-1049, 2001.

12. Slomka PJ, Mandel J, Downey D, Fenster A: Evaluation of voxel-based registration of 3-D power Doppler ultrasound and 3-D magnetic resonance angiographic images of carotid arteries. Ultrasound Med Biol 27(7):945-955, 2001.

13. Pennec X, Cachier P, Ayache N: Tracking brain deformations in time sequences of 3D US images. Pattern Recognition Letters 24(4-5):801-813, 2003.

14. Letteboer M, Willems P, Viergever MA, Niessen W: Non-rigid Registration of 3D Ultrasound Images of Brain Tumours Acquired during Neurosurgery. MICCAI 2003, LNCS 2879:408-415, 2003.

15. Porter BC, Rubens DJ, Strang JG, et al.: Three-Dimensional Registration and Fusion of Ultrasound and MRI Using Major Vessels as Fiducial Markers. IEEE Trans Med Imag 20(4):354-359, 2001.

16. Penney GP, Blackall JM, Hayashi D, et al.: Overview of an ultrasound to CT or MR registration system for use in thermal ablation of liver metastases. Proc. Medical Image Understanding and Analysis, 2001.

17. Aylward SR, Jomier J, Weeks S, Bullitt E: Registration and Analysis of Vascular Images. Int J Computer Vision 55(2-3):123-138, 2003.

18. Xie Z, Farin GE: Deformation With Hierachical B-Splines. Mathematical Methods in Computer Aided Geometric Design, 545-554, 2001.

19. Sato M, Bitter I, Bende M, et al.: TEASAR: Tree-structure extraction algorithm for accurate and robust skeletons. Procs. PACIFIC GRAPHICS-00, 281-289, 2000.

20. Cash DM, Sinha TK, Chapman WC, et al.: Incorporation of a laser range scanner into image-guided liver surgery: surface acquisition, registration, and tracking. Medical Physics 30(7):1671-1682, 2003. 